\title{
The effects of a diet enriched with $\alpha$-lactalbumin on mood and cortisol response in unmedicated recovered depressed subjects and controls
}

\author{
Wendelien Merens ${ }^{1}$, Linda Booij ${ }^{1}$, Rob Markus ${ }^{2}$, Frans G. Zitman ${ }^{3}$, Willem Onkenhout ${ }^{4}$ \\ and A.J. Willem Van der Does ${ }^{1,3 *}$ \\ ${ }^{1}$ Department of Psychology, Leiden University, Leiden, The Netherlands \\ ${ }^{2}$ Department of Psychology, Maastricht University, Maastricht, The Netherlands \\ ${ }^{3}$ Department of Psychiatry, Leiden University Medical Center, Leiden, The Netherlands \\ ${ }^{4}$ Department of Pediatrics, Leiden University Medical Center, Leiden, The Netherlands
}

(Received 12 November 2004 - Revised 22 March 2005 - Accepted 30 March 2005)

\begin{abstract}
$\alpha$-Lactalbumin is a tryptophan-rich protein fraction. A diet enriched with $\alpha$-lactalbumin increases the ratio of tryptophan to the other large neutral amino acids, which may in turn increase brain serotonin content. In stress-vulnerable individuals, $\alpha$-lactalbumin improved mood and attenuated the cortisol response after experimental stress. The aim of the present study was to investigate the effects of an $\alpha$-lactalbumin-enriched diet on mood and stress response in recovered depressed subjects and healthy controls. Forty-three subjects (twenty-three recovered depressed and twenty healthy subjects) received $\alpha$-lactalbumin and casein (placebo) on separate days, in a double-blind randomised crossover design. On both occasions, subjects underwent a stress test (an unsolvable mental arithmetic task with loud noise). The stress test affected mood in both conditions. Although the $\alpha$-lactalbumin diet led to the expected rises in tryptophan and tryptophan:large neutral amino acids ratio, only minimal effects were found on mood and cortisol response to experimental stress. The results were the same for recovered depressed patients and controls. A $1 \mathrm{~d}$ diet enriched with $\alpha$-lactalbumin is not sufficient to prevent a stress-induced mood deterioration or a cortisol response in unmedicated, recovered depressed subjects. Future studies may investigate the effects of longer-term diets or may investigate different samples (e.g. medicated patients).
\end{abstract}

$\alpha$-Lactalbumin: Depression: Serotonin: Cortisol

There is abundant evidence that serotonin (5-hydroxytryptamine; 5-HT) plays an important role in stress-related disorders such as major depression (Meltzer \& Lowy, 1987; Maes \& Meltzer, 1995). For instance, medications that augment 5-HT activity, such as selective serotonin reuptake inhibitors and monoamine oxidase inhibitors, are effective antidepressants (Blier \& De Montigny, 1998; Nutt et al. 1999). Furthermore, experimental depletion of the 5-HT precursor L-tryptophan (Trp) induces symptoms in depression-vulnerable subjects (patients in remission or family members of patients) but not in healthy subjects (Bell et al. 2001; Van der Does, 2001). Conversely, Trp administration increases 5-HT synthesis in the brain in both human subjects and rats, and these effects are large enough to influence mood and behaviour (Young, 1996). For example, Trp decreased aggression and quarrelsome behaviour, and increased dominant behaviour, in healthy volunteers (Moskowitz et al. 2001; Marsh et al. 2002; Young \& Leyton, 2002). In healthy females, Trp improved emotion recognition (Attenburrow et al. 2003). Trp also has some therapeutic effect in mild to moderate depression, but it is not effective in more severe depression (Young, 1986, 1996). It is clear, however, that boosting or depleting the 5-HT system has opposite effects on mood, although some of these effects are only observable in depression-vulnerable subjects.
Several studies have attempted to increase 5-HT concentrations through dietary interventions. Although selective serotonin reuptake inhibitors have fewer side-effects than other antidepressants, and are not toxic in overdose, a sizable number of patients do not tolerate them or fail to benefit from them (Fava, 2000). Survey data show that many patients prematurely stop their medications because of side-effects (Consumer Reports, 2004). So, if effective and feasible, dietary interventions may comprise a viable addition or alternative to antidepressant medications. A carbohydrate-rich/protein-poor diet has been shown to increase the ratio of Trp:large neutral amino acids (LNAA). The diet prevented stress-induced mood deterioration and cortisol response in individuals vulnerable to stress (defined by high neuroticism scores; Markus et al., 1998). Carbohydrate-rich/proteinpoor diets are, however, not healthy on a day-to-day basis (Cristensen, 1997), and even a small amount of protein blocks the rise of Trp:LNAA.

Recently, an even larger effect on stress response was obtained with a diet containing Trp-rich $\alpha$-lactalbumin protein. $\alpha$-Lactalbumin has the highest Trp concentration of all protein fractions (Heine et al. 1996). This diet increased plasma Trp:LNAA by $43-48 \%$, which is twice the augmentation found with a carbohydrate-rich/protein-poor diet (Markus et al. 2000, 2002), or after

Abbreviations: BDI-II, Beck Depression Inventory; DSC, Depressed States Checklist; DSM, Diagnostic and Statistical Manual of Mental Disorders-IV; 5-HT, 5-hydroxytryptamine; LNAA, large neutral amino acids; MADRS, Montgomery Asberg Depression Rating Scale; POMS, Profile of Mood States; Trp, L-tryptophan. * Corresponding author: Dr A. J. W. Van der Does, fax +31 71527 3619, email vanderdoes@fsw.leidenuniv.nl 
$7 \mathrm{~d}$ of daily Trp treatment (Chouinard et al. 1985). $\alpha$-Lactalbumin had no side-effects, and improved mood and attenuated cortisol responses to stress in stress-vulnerable subjects (students with high neuroticism scores) but not in controls (low neuroticism; Markus et al. 2000). Orosco et al. (2004) found that $\alpha$-lactalbumin had an anxiolytic effect in rats and increased 5-HT turnover in hippocampal areas. Since depression-vulnerable individuals are most likely to be affected by 5 -HT manipulations, it seems worthwhile exploring the effects of $\alpha$-lactalbumin in this group.

The present study was designed to investigate the effects of $\alpha$-lactalbumin in depression-vulnerable individuals and in healthy, non-vulnerable controls. Based on previous research (Markus et al. 2000,2002 ), we expected to find a protective effect of $\alpha$-lactalbumin on stress-induced mood deterioration and cortisol response, rather than a direct effect on mood. Furthermore, we expected these effects to occur only in the depression-vulnerable group.

\section{Materials and methods}

\section{Subjects}

Eligible subjects were between 18 and 65 years of age and had either a history of a major depressive episode (patient group) or no history of mental disorder and no first-degree relative with a major depressive disorder (control group). Subjects were euthymic and were selected not on the basis of neuroticism scores (Markus et al. 2000, 2002) but on a history of depression. Approximately $60 \%$ of people with high neuroticism scores actually develop a depression (Gallagher, 1990; Ormel et al. 2001; Kendler et al. 2004). Since long-term studies have shown that up to $85 \%$ of recovered depressed patients experience a recurrence (Mueller et al. 1999), a past history of depression is a better marker of vulnerability.

In both groups, exclusion criteria were: current mental disorder; past psychotic disorder; substance abuse in the past 3 months or excessive dieting or binge eating, as defined by the Diagnostic and Statistical Manual of Mental Disorders-IV (DSM-IV; American Psychiatric Association, 1994). Furthermore, subjects in both groups had low levels of depressive symptoms (Montgomery Asberg Depression Rating Scale (MADRS) score $<8$, were not colour-blind or dyslexic, had been free of antidepressant medication for at least 3 months, had a normal body weight (BMI $18-27 \mathrm{~kg} / \mathrm{m}^{2}$ ), kept to a regular diet and were free of neuroendocrine or neurological disease. Female subjects were not pregnant or lactating, had a regular menstrual cycle or were postmenopausal. Premenopausal women who were not taking a contraceptive pill were tested during their mid-to-late follicular phase (days 4-10), whereas premenopausal women taking a contraceptive pill participated during the period in which they actually took the pill. The groups were matched on gender, age and education level.

\section{Design}

The study was conducted according to a double-blind crossover design. Subjects were given balanced isoenergetic meals containing $\alpha$-lactalbumin-enriched whey protein or casein protein (placebo). Treatment order was balanced over two sessions, separated by 4 weeks.

\section{Instruments}

Standardised, validated instruments were used to assess diagnosis, depressive symptoms, mood states and personality. The Structured Clinical Interview for DSM-IV (First et al. 1995) was used to verify diagnostic inclusion and exclusion criteria. Depressive symptoms were measured with the MADRS (Montgomery \& Asberg, 1979), a ten-item interviewer-rating scale, and the self-rating Beck Depression Inventory (BDI-II; Beck et al. 1996; Van der Does, 2002a).

The Profile of Mood States (POMS; McNair et al. 1971; Wald \& Mellenbergh, 1990) measures 'depression', 'anger', 'fatigue', 'tension' and 'vigor'. Neuroticism was measured to facilitate comparison with previous research (Markus et al. 2000, 2002); the Eysenck Personality Questionnaire (Eysenck et al. 1985) was used. Cognitive reactivity, a psychological vulnerability marker of depression, was also measured, using the Depressed States Checklist (DSC; Teasdale \& Cox, 2001) and the Leiden Index of Depression Sensitivity (Van der Does, 2002b). The DSC consists of items that 'objectively' describe a depressed mood (e.g. sad, unhappy), and items that also have a self-devaluative denotation (e.g. useless, a failure). Cognitive reactivity is the ratio of the endorsed self-devaluative and objective items (Teasdale \& Cox, 2001). The Leiden Index of Depression Sensitivity is a self-rating scale of cognitive reactivity, with subscales 'hopelessness', 'rumination', 'harm avoidance', 'aggression', 'control/perfectionism' and 'acceptance/coping' (Van der Does, 2002b).

\section{Procedure}

Depressed patients who had participated in a psychotherapy trial 1-2 years earlier were sent information and invited to participate. Subjects were also recruited through advertisements in a local newspaper, and through advertisements and leaflets at the local mental health centre and at several university buildings. Individuals who expressed interest were interviewed by telephone. If the inclusion and exclusion criteria appeared to be met, more information was sent, and people were invited for a screening session at the hospital, during which the Structured Clinical Interview for DSM-IV and MADRS was administered by trained clinical psychologists. Information about previous antidepressant treatments and the course and duration of depressive episodes was also obtained, and questionnaires were completed. Subjects gave informed consent to participate prior to filling out the questionnaires. The first session was scheduled for approximately 1 week after the screening session, or somewhat later depending on the menstrual cycle phase.

Subjects arrived at the laboratory at 09.00 hours after fasting overnight. They were instructed not to use alcohol for $24 \mathrm{~h}$ prior to the sessions and to arrive well rested. They received breakfast and lunch including either $\alpha$-lactalbumin or casein. In the morning and afternoon, a battery of cognitive tests was administered, the results of which are reported elsewhere (Booij et al. 2005). During the day, two blood samples and four cortisol samples were taken. The session finished at around 15.30 hours, and subjects were instructed to resume their regular meals. The outline of sessions is shown in Table 1. Subjects were paid $€ 80$ for participation. Subjects were tested individually at a clinical research unit of Leiden University Medical Center. 


\section{Intervention}

The $\alpha$-lactalbumin and casein diets used in the present study were identical to the diets used by Markus et al. (2000, 2002). Diets contained $1229 \mathrm{~kJ}$ with $5 \cdot 2 \%$ energy as protein, $84.2 \%$ energy as carbohydrate and $10.6 \%$ energy as fat, composed of standard products (Netherlands Nutrition Center). Each breakfast and lunch consisted of one slice of bread, $10 \mathrm{~g}$ butter, $15 \mathrm{~g}$ fruit jelly, $200 \mathrm{ml}$ grape juice and a chocolate drink. The two diets were similar in appearance and in macronutrient content with the exception of the composition of the chocolate-flavoured drink, for which the protein sources differed. Apart from breakfast and lunch, subjects were only allowed to drink water and one cup of tea or coffee.

The nutrient composition and amino acid profile of both chocolate drinks are displayed in Table 2. The amounts of Trp in the $\alpha$-lactalbumin and casein diets were $12.32 \mathrm{~g} / \mathrm{kg}$ (Trp:LNAA 8.7) and $9.51 \mathrm{~g} / \mathrm{kg}$ (Trp:LNAA 4.7), respectively. The chocolate drink was prepared within $20 \mathrm{~min}$ before breakfast (first drink) and $20 \mathrm{~min}$ before lunch (second drink). To compensate for any taste differences, sugar was added to all the drinks ( $10 \mathrm{~g}$; see Table 2$)$. All meals were supervised to make sure that all foods were consumed.

\section{Stress task}

An impossible mental arithmetic task, performed under noise stimulation, was used as an experimental stressor. Subjects were given eighteen successive 1 min trials, during which they had to do mental arithmetic under time constraints, while different levels of industrial noise $(65,70$ or $80 \mathrm{~dB})$ were presented through a headphone. Multiple choice questions were presented on a computer screen one at a time. A specified number of calculations (called the criterion) had to be solved correctly. Subjects were led to believe that they could control the intensity of noise by their performance. If they failed the criterion, the computer would set the noise level higher during the next trial; if, however, they met the criterion, they could choose the noise level themselves. Before the actual test, subjects were given two practice trials during which they had to solve a few calculations without noise and then with each of the three noise levels successively. The credibility of the task as well as motivation were enhanced by providing the subjects with constant on-screen feedback about the criterion in a particular trial, the number of calculations correctly solved and the time left for that trial.

Experimental stress was induced by manipulating the criterion so that all subjects continued to fail on each trial and thus could not choose the noise level for the next trial. The criterion was

Table 1. Scheme of the sessions

\begin{tabular}{lll}
\hline Time & \multicolumn{2}{l}{ Assessment } \\
\hline 09.00 hours & $t_{-1}$ & Arrival, BDI-II, DSC, POMS, blood sample \\
10.00 hours & $t_{0}$ & Breakfast \\
12.00 hours & $t_{2}$ & Lunch \\
13.30 hours & $t_{3.5}$ & Cortisol (1), blood sample, POMS, DSC \\
14.35 hours & $t_{4.5}$ & Cortisol (2), POMS \\
14.40 hours & & Stress induction \\
15.00 hours & $t_{5}$ & Cortisol (3), POMS, DSC \\
15.10 hours & $t_{5.2}$ & Cortisol (4), debriefing
\end{tabular}

BDI-II, Beck Depression Inventory-II; DSC, Depressed States Checklist; POMS, Profile o Mood States.

For details of diets and procedures, see p. 416
Table 2. Composition of the chocolate drinks used in the $\alpha$-lactalbumin and casein diets

\begin{tabular}{lcc}
\hline & $\alpha$-Lactalbumin diet & Casein diet \\
\hline Composition (g) & & \\
$\quad \alpha$-Lactalbumin-enriched whey protein & 20 & 0 \\
Sodium caseinate & 0 & 20 \\
Cocoa & 3.5 & 3.5 \\
Granulated sugar & 10 & 10 \\
Water & 200 & 200 \\
Amino acid profile (g/kg) & & \\
Isoleucine & 27.61 & 31.80 \\
Leucine & 47.56 & 59.31 \\
Phenylalanine & 20.80 & 32.24 \\
Tyrosine & 16.82 & 33.13 \\
Valine & 29.52 & 44.09 \\
Tryptophan & 12.32 & 9.51 \\
Tryptophan:large neutral amino acids & 8.70 & 4.70 \\
$\quad$ (weight \%) & & \\
\end{tabular}

For details of diets and procedures, see p. 416.

always set at one sum above what subjects could manage, as calculated from the average time per sum needed on the previous trial. This task has been demonstrated to be uncontrollable and to induce transient psychological and physiological stress (Peters et al. 1998; Markus et al. 1999, 2000, 2002). The stress task proved to be sensitive in crossover designs in studies by Markus et al. In addition, the test affects perceived stress as well as systolic and diastolic blood pressure (Peters et al. 1998).

\section{Salivary cortisol}

Cortisol samples were obtained with the Salivette sampling device (Sarstedt, Nümbrecht, Germany). With this procedure, saliva was collected in small cotton swabs and stored in special Salivette tubes at $-30^{\circ} \mathrm{C}$ until centrifugation. Saliva samples were centrifuged at $3000 \mathrm{rpm}$ for $3 \mathrm{~min}$ at $20^{\circ} \mathrm{C}$. Cortisol concentrations were determined without extraction using a competitive chemiluminescence immunoassay.

A $20 \mu \mathrm{l}$ sample of saliva was pipetted into microtitre plate wells coated with rabbit anti-cortisol antibodies. After the addition of $100 \mu \mathrm{l}$ cortisol conjugated with horseradish peroxidase (enzyme conjugate), the mixture was incubated for $3 \mathrm{~h}$ at room temperature, and the cells were washed to stop the competition reaction. After addition of the chemiluminescence substrate solution containing peroxide and luminol, luminescence measurements were performed using a Berthold microtitre plate luminometer (Medical Laboratories, Mönchengladbach, Germany).

\section{Blood assessments}

Total Trp and its concentration ratio to LNAA (valine, isoleucine, leucine, tyrosine and phenylalanine) were determined by amino acid analysis. Blood was collected in $4 \mathrm{ml}$ EDTA-containing tubes, and plasma was isolated after centrifugation at $2650 \mathrm{~g}$ at $20^{\circ} \mathrm{C}$ for $20 \mathrm{~min}$. For the determination of (total) Trp and LNAA, $400 \mu \mathrm{l}$ plasma was deproteinised using an equal volume of $0.075(\mathrm{w} / \mathrm{v})$ sulphosalicylic acid in water, which concomitantly contained $382 \mu \mathrm{mol} / \mathrm{l}$ of the internal standard L-2,4-diamino-butyric acid (Fluka, Milan, Italy). The supernatant was isolated after centrifugation at $8000 \mathrm{~g}$ at $20^{\circ} \mathrm{C}$ for $2 \mathrm{~min}$, filtered through Millex $0.22 \mu \mathrm{m}, 25 \mathrm{~mm}$ membrane filter units (Millipore, Billerica, MA, USA); $60 \mu \mathrm{l}$ of this was analysed in $170 \mathrm{~min}$ by 
means of ion-exchange chromatography and ninhydrin derivatisation on a Biochrom 20 automated amino acid analyser (Pharmacia, Uppsala, Sweden) using standard conditions for physiological amino acid separation.

\section{Statistical analyses}

Group differences in demographic characteristics were examined with independent-sample $t$ tests. The effects of the interventions on cortisol response were analysed by means of repeated-measures ANOVA using general linear models. Within-subjects factors were intervention ( $\alpha$-lactalbumin $v$. casein) and time of assessment (before and after stress); the between-subjects factor was group (recovered depressed $v$. controls). All mood scores were analysed using non-parametric tests (Wilcoxon and Mann-Whitney U). A test was considered to be significant when the $P$ value was 0.05 or less. SPSS 11.5 (SPSS Inc., Chicago IL, USA) was used.

\section{Ethical considerations}

The study was approved by the Medical Ethics Committee of Leiden University Medical Center. Previous studies using casein or $\alpha$-lactalbumin diets have shown that the procedure is safe, with no side-effects (Markus et al. 2000, 2002). Prior studies have used the stress induction task (Peters et al. 1998; Markus et al. 1999, 2000, 2002). There are no indications that the cognitive tasks or the stress task elicit the recurrence of depression.

\section{Results}

\section{Data screening}

A very small number of missing values on items of the symptom questionnaires were replaced by the grand mean. No statistical outliers were detected. Twenty-two blood samples (13\% of the total) were not available owing to difficulties with venepuncture. The amino acid levels and ratios were all normally distributed. Cortisol values and the scores on the mood subscales were not; these data were ${ }^{10} \log$-transformed. Subscales of the POMS and DSC were analysed using non-parametric tests as the data were highly skewed and transformations were unsuccessful.

\section{Sample characteristics}

Fifty-seven individuals were invited for the screening session, eight of whom did not meet the inclusion and exclusion criteria. Three eligible subjects dropped out after the screening session, and another three dropped out after the first test day for various reasons. A total of forty-three subjects completed both test days. As shown in Table 3, there were no significant differences in age, education and gender distribution between groups. MADRS scores in both groups were low and did not differ significantly. The only significant difference was found on BDIII scores at screening, with recovered depressed subjects having higher scores. However, BDI-II scores were low and well within the normal range in both groups. As shown in Table 4, the recovered depressed patient group had a mean age of depression onset at 19.9 years. The mean number of episodes was two. Approximately one-third had taken antidepressant medication in the past, and almost half had a first-degree relative with depression.

Significant baseline differences between the groups were found on the POMS depression $(Z-2 \cdot 21, P=0 \cdot 03)$, POMS fatigue $(Z-2 \cdot 06, P=0 \cdot 04)$, POMS total $(Z-2 \cdot 31, P=0 \cdot 02)$ and DSC self-devaluative $(Z-2.42, P=0.02)$ subscales. Trends were found for POMS tension $(Z-1.77, P=0.08)$ and DSC affective ( $Z-1.79, P=0.07)$. The recovered depressed subjects had

Table 3. Demographic and clinical characteristics

(Mean values and standard deviations)

\begin{tabular}{|c|c|c|c|c|c|c|c|}
\hline & \multicolumn{2}{|c|}{$\begin{array}{c}\text { Recovered } \\
\text { depressive (n 23) }\end{array}$} & \multicolumn{2}{|c|}{$\begin{array}{l}\text { Healthy controls } \\
\qquad(n 20)\end{array}$} & \multicolumn{3}{|c|}{ Recovered $v$. controls } \\
\hline & Mean & SD & Mean & SD & $t / Z$ & df & $P$ \\
\hline Age & $30 \cdot 0$ & $9 \cdot 7$ & $27 \cdot 0$ & $10 \cdot 1$ & 0.99 & 41 & 0.32 \\
\hline Female (\%) & $91 \cdot 3$ & & 85 & & $\left(\chi^{2}\right) 0.41$ & 1 & 0.52 \\
\hline Educational level ${ }^{*}$ & 4.9 & 0.8 & $5 \cdot 1$ & 0.6 & -1.07 & 41 & 0.29 \\
\hline BMI $\left(\mathrm{kg} / \mathrm{m}^{2}\right)$ & $22 \cdot 8$ & $2 \cdot 5$ & $21 \cdot 7$ & $2 \cdot 1$ & 1.60 & 41 & 0.12 \\
\hline MADRS & $1 \cdot 3$ & 1.6 & 0.8 & 1.6 & 1.03 & 41 & 0.31 \\
\hline BDI-II & 4.4 & 4.5 & 1.5 & $2 \cdot 2$ & $2 \cdot 83$ & 41 & 0.008 \\
\hline EPQ-R psychoticism & 3.2 & $1 \cdot 7$ & $2 \cdot 6$ & $1 \cdot 3$ & $1 \cdot 29$ & 41 & 0.20 \\
\hline Extraversion & $7 \cdot 6$ & 3.4 & 9.7 & $2 \cdot 3$ & $-2 \cdot 31$ & 41 & 0.03 \\
\hline Neuroticism & $5 \cdot 6$ & $2 \cdot 3$ & $2 \cdot 6$ & $2 \cdot 0$ & 4.43 & 41 & $<0.001$ \\
\hline Social desirableness & $5 \cdot 7$ & $2 \cdot 1$ & $5 \cdot 8$ & $2 \cdot 1$ & -0.10 & 41 & 0.92 \\
\hline LEIDS hopelessness & $4 \cdot 6$ & 3.0 & $1 \cdot 4$ & 1.0 & $4 \cdot 76$ & 41 & $<0.001$ \\
\hline Aggression & $5 \cdot 8$ & 3.9 & $3 \cdot 1$ & $3 \cdot 1$ & 2.45 & 41 & 0.02 \\
\hline Perfectionism & 8.0 & $4 \cdot 7$ & $4 \cdot 1$ & $3 \cdot 3$ & $3 \cdot 18$ & 41 & 0.003 \\
\hline Harm avoidance & 9.5 & $4 \cdot 8$ & 3.5 & $2 \cdot 6$ & $5 \cdot 23$ & 41 & $<0.001$ \\
\hline Rumination & $10 \cdot 7$ & 4.9 & $5 \cdot 6$ & $2 \cdot 5$ & $4 \cdot 39$ & 41 & $<0.001$ \\
\hline Acceptance/coping & $2 \cdot 2$ & 3.9 & 1.0 & $1 \cdot 7$ & 1.38 & 41 & 0.18 \\
\hline Total score & $40 \cdot 8$ & $18 \cdot 9$ & $18 \cdot 5$ & $9 \cdot 8$ & 4.94 & 41 & $<0.001$ \\
\hline DSC affective & $26 \cdot 5$ & $9 \cdot 8$ & $21 \cdot 1$ & $5 \cdot 0$ & 1.79 & & 0.07 \\
\hline Self-devaluative & $22 \cdot 0$ & $6 \cdot 6$ & $17 \cdot 0$ & $2 \cdot 6$ & $2 \cdot 42$ & & 0.02 \\
\hline
\end{tabular}

MADRS, Montgomery-Asberg Depression Rating Scale; BDI-II, Beck Depression Inventory-II; EPQ-R, Eysenck Personality Questionnaire-Revised; LEIDS, Leiden Index of Depression Sensitivity; DSC, Depressed States Checklist.

* On a scale of 1-6.

For details of subjects and procedures, see p. 416. 
Table 4. Clinical characteristics of the recovered depressed subjects

\begin{tabular}{|c|c|c|c|}
\hline & Mean & & SD \\
\hline Age of onset (years) & $19 \cdot 9$ & & $7 \cdot 7$ \\
\hline Number of episodes & $2 \cdot 0$ & & 0.9 \\
\hline Antidepressant medication (past) & & $34.8 \%$ & \\
\hline Antidepressant treatment (ever) & & $78.3 \%$ & \\
\hline $\begin{array}{l}\text { Family history (first degree) } \\
\text { of major depressive disorder }\end{array}$ & & $47 \cdot 8 \%$ & \\
\hline Current psychotherapy & & $13 \%$ & \\
\hline
\end{tabular}

For details of subjects and procedures, see p. 416.

higher scores on all these subscales. On the Eysenck Personality Questionnaire, groups differed on extraversion $(F(1,41) 5 \cdot 32$, $P=0.03)$ and neuroticism $(F(1,41) 19.6, P<0.001)$. The neuroticism scores of the recovered depressed group were above average to high, according to published norms for Dutch populations (Sanderman et al. 1995). The two groups also differed on most subscales of the Leiden Index of Depression Sensitivity. After controlling for depressive symptoms (BDI-II), only the following subscales remained significant: neuroticism $(F(1,40)$ 12.9, $P=0 \cdot 001$ ), and the Leiden Index of Depression Sensitivity subscales of hopelessness $(F(1,40) 13 \cdot 6, P=0 \cdot 001)$, control/perfectionism $(F(1,40) 5 \cdot 6, P=0 \cdot 02)$, harm avoidance $(F(1,40) 16 \cdot 5$, $P<0.001)$, rumination $(F(1,40) \quad 10.5, \quad P=0.002)$ and total $(F(1,40) 13 \cdot 9, P=0 \cdot 001)$.

\section{Effect of dietary manipulations on amino acids}

As shown in Table 5, plasma Trp concentrations and Trp:LNAA did not differ at baseline between groups. Repeated-measures analyses with intervention and time as within-subjects factors,

Table 5. Plasma tryptophan $(\mu \mathrm{mol} / \mathrm{l})$ and tryptophan:large neutral amino acid (Trp:LNAA ratio)

(Mean values and standard deviations)

\begin{tabular}{llccccc}
\hline & \multicolumn{2}{c}{ Casein } & & \multicolumn{2}{c}{$\alpha$-Lactalbumin } \\
\cline { 3 - 4 } & & Mean & SD & & Mean & SD \\
\hline \multirow{2}{*}{ Tryptophan } & Morning & 50.5 & 11.2 & & 50.8 & 10.1 \\
& Afternoon & 57.3 & 8.9 & & 87.9 & 14.5 \\
Trp/LNAA & Morning & 0.11 & 0.02 & & 0.11 & 0.02 \\
& Afternoon & 0.08 & 0.01 & & 0.13 & 0.02 \\
& & & & & &
\end{tabular}

For details of diets and procedures, see p. 416 . and group as the between-subjects factor, on Trp:LNAA revealed a significant effect of intervention $(F(1,29)$ 84.3, $P<0 \cdot 001)$. Trp:LNAA increased significantly by $20.9 \%$ compared with baseline after the $\alpha$-lactalbumin diet and decreased by $30.0 \%$ after the casein diet. The relative rise of Trp:LNAA was $73.8 \%$ higher after $\alpha$-lactalbumin than after casein. We also found significant effects of time $(F(1,29) 7 \cdot 1, P=0.013)$ and of the intervention $\times$ time interaction $(F(1,29) 193.3, P<0.001)$. There was no main effect of group, nor any interaction effects involving group.

\section{Effects of stress on cortisol}

Two cortisol samples were collected after the stress task, $t_{5}$ (directly after the stress) and $t_{5 \cdot 2}(10$ min later). The higher cortisol level of these two was used in the analyses $\left(t_{\max }\right)$. Repeatedmeasures analyses with intervention and time (before and after stress) as within-subjects factors, and group as the betweensubjects factor, on cortisol level did not reveal the expected interaction of intervention $\times$ group $\times$ time $(F(1,41) \quad 0 \cdot 1, P=0 \cdot 71)$. We did, however, find a significant main effect of time $(F(1,41)$ 9.7, $P=0.003)$, reflecting an increase in cortisol level after stress. No other main or interaction effects were found. Cortisol levels are shown in Table 6.

To check for any order of intervention effects, order, instead of group, was included in the analysis as a between-subjects factor. No interaction effect of order $\times$ intervention was found $(F(1,41)$ $0.01, P=0.93)$. A trend for an interaction effect of intervention $\times$ time $\times$ order was found $(F(1,41) 3 \cdot 4, P=0 \cdot 07)$, caused by the fact that cortisol did not rise after stress for subjects who had the $\alpha$ lactalbumin diet on the second day. $\alpha$-Lactalbumin did not have this protective effect for subjects who had that diet on the first day.

Although Markus et al. (1998, 2000) found cortisol responses in both men and women - using the same stressor - previous research with other stressors has shown that response may be dependent on gender, and for females on menstrual cycle and use of oral contraceptives (Kirschbaum et al. 1999). We therefore divided the total group of forty-three subjects into three groups: men ( $n$ 5), women taking ( $n$ 23) and women not taking ( $n$ 15) hormonal contraceptives. This analysis showed significant interaction effects of intervention $\times$ group $(F(2,41) 4.3, P=0.02)$, and of time $\times$ group $(F(2,41) 3 \cdot 8, P=0 \cdot 03)$. Women taking contraceptives had no cortisol response to stress. Women not taking hormones had a small rise of cortisol on the casein day and not on the $\alpha$-lactalbumin day, whereas men showed the reverse pattern. Because of the small groups and the opposite effects in men and women, no further analyses were carried out.

Table 6. Cortisol (nmol/l) before $\left(t_{4.5}\right)$ and after $\left(t_{\max }\right)$ stress (Mean values and standard deviations)

\begin{tabular}{|c|c|c|c|c|c|c|c|c|}
\hline & \multicolumn{4}{|c|}{ Casein } & \multicolumn{4}{|c|}{$\alpha$-Lactalbumin } \\
\hline & \multicolumn{2}{|c|}{ Before stress } & \multicolumn{2}{|c|}{ After stress } & \multicolumn{2}{|c|}{ Before stress } & \multicolumn{2}{|c|}{ After stress } \\
\hline & Mean & SD & Mean & SD & Mean & SD & Mean & SD \\
\hline Healthy controls ( $n 20)$ & $5 \cdot 1$ & $6 \cdot 4$ & $5 \cdot 9$ & $6 \cdot 3$ & $5 \cdot 7$ & $5 \cdot 1$ & $5 \cdot 4$ & $2 \cdot 8$ \\
\hline Recovered depressed subjects ( $n$ 23) & $5 \cdot 2$ & $3 \cdot 8$ & $7 \cdot 4$ & $7 \cdot 0$ & 4.5 & 1.8 & $6 \cdot 4$ & 6.4 \\
\hline High-neuroticism subjects ( $n$ 27) & $5 \cdot 1$ & 3.7 & $6 \cdot 8$ & $6 \cdot 6$ & $4 \cdot 2$ & $2 \cdot 0$ & $5 \cdot 0$ & $4 \cdot 1$ \\
\hline Low-neuroticism subjects ( $n$ 16) & $5 \cdot 4$ & $7 \cdot 1$ & 6.5 & $7 \cdot 0$ & 6.5 & $5 \cdot 3$ & $7 \cdot 6$ & $6 \cdot 1$ \\
\hline
\end{tabular}

For details of subjects and procedures, see p. 416. 


\section{Effects of $\alpha$-lactalbumin on mood}

To investigate any direct effects of $\alpha$-lactalbumin on mood, we analysed the differences in mood between the morning $\left(t_{-1}\right)$ and the first afternoon rating $\left(t_{3 \cdot 5}\right)$. On the $\alpha$-lactalbumin day, the POMS tension $(Z-2 \cdot 6, P=0 \cdot 008)$ and DSC self-devaluative $(Z-2 \cdot 0, P=0 \cdot 04)$ ratings decreased. A trend was found for POMS anger $(Z-1.9$, $P=0.06)$. However, on the casein day, POMS anger $(Z 2 \cdot 9$, $P=0.003)$, depression $(Z-2 \cdot 8, P=0.005)$ and fatigue $(Z-2 \cdot 1$, $P=0.035)$, as well as both DSC scores, decreased. During the $\alpha$-lactalbumin session, recovered patients had (almost) significantly larger decreases in DSC self-devaluative $(Z-2 \cdot 2, P=0.03)$ and POMS depression $(Z-1.9, P=0.06)$ scores than controls. The difference in self-devaluative change scores between groups was also significant in the casein condition $(Z-2 \cdot 2, P=0 \cdot 03)$. No other group differences were observed. Thus, mood improved slightly after both interventions. Only POMS depression scores tended to improve more in the recovered depressed group than in the control group on the $\alpha$-lactalbumin day. However, this effect was due to a floor effect in the control group. So, as hypothesised, no direct effects of $\alpha$-lactalbumin on mood were found.

\section{Effects of stress on mood}

To investigate any protective effects of $\alpha$-lactalbumin on the response to stress, scores on the POMS and DSC scales before and after stress were analysed. Mood scores are shown in Table 7. In both conditions, significant increases in DSC affective and self-devaluative scores were observed. In the $\alpha$-lactalbumin condition, POMS anger, tension, depression and total scores increased, fatigue tended to increase and vigor decreased in response to stress. In the casein condition, similar changes were found for POMS anger, tension and total scores. A trend was found for depression in the casein condition. In the $\alpha$-lactalbumin condition, recovered depressed subjects showed a significantly larger increase in self-devaluative scores compared with controls $(Z-2 \cdot 0, P=0 \cdot 04)$. There were no differences between groups in POMS change scores in response to stress.

To analyse the effect of order on mood response to stress, Kruskal-Wallis tests were performed on mood change scores with order as the grouping variable. A significant effect of order on vigor change score was found in both the recovered depressed group ( $\left.\chi^{2} 5 \cdot 8, P=0.016\right)$ and the control group ( $\left.\chi^{2} 4 \cdot 3, P=0.037\right)$, but only for casein. The decrease of vigor was smaller if casein was given on day 2 , suggesting that the stressful nature of the task might have been slightly reduced on the second day. The same effect was found for the DSC, but then only in the controls. No other effects of order were found.

Results therefore showed that the stressor affected mood in both the $\alpha$-lactalbumin and the casein condition. The effects were, however, much smaller than expected, and there were no differences between the two diets.

To explore the reasons for these unexpected findings, we also looked at possible effects of past antidepressant medication use, family history of depression and cognitive reactivity on cortisol response and on mood. Again, only significant effects of time were found. Previous research (Markus et al. 2000) using the same paradigm in a healthy population divided on the basis of neuroticism scores found effects of $\alpha$-lactalbumin on mood and cortisol response. Therefore, we reallocated subjects to high- and low-neuroticism groups (median split; see Table 6). As in the previous analyses contrasting previously depressed and never-depressed subjects, no between-group differences in cortisol response were found. A significant interaction effect of intervention $\times$ group $(F(1,41) 7 \cdot 9, P=0 \cdot 007)$ and a main effect of time $(F(1,41) 10 \cdot 3$, $P=0.003)$ were again found. Further analyses indicated that the low-neuroticism group had higher cortisol levels on the $\alpha$-lactalbumin day than on the casein day and that both groups showed an increase in cortisol level after stress.

Table 7. Mood before and after experimental stress

(Mean values and standard deviations)

\begin{tabular}{|c|c|c|c|c|c|c|c|c|}
\hline & \multicolumn{4}{|c|}{ Casein diet } & \multicolumn{4}{|c|}{$\alpha$-Lactalbumin diet } \\
\hline & \multicolumn{2}{|c|}{ Before stress } & \multicolumn{2}{|c|}{ After stress } & \multicolumn{2}{|c|}{ Before stress } & \multicolumn{2}{|c|}{ After stress } \\
\hline & Mean & SD & Mean & SD & Mean & $\mathrm{SD}$ & Mean & SD \\
\hline \multicolumn{9}{|l|}{ Healthy controls ( $n 20)$} \\
\hline \multicolumn{9}{|l|}{ POMS } \\
\hline Anger & $7 \cdot 2$ & 0.4 & $8 \cdot 4$ & $1 \cdot 8$ & $7 \cdot 4$ & $1 \cdot 1$ & 8.6 & $1 \cdot 8$ \\
\hline Tension & $5 \cdot 4$ & 0.7 & $5 \cdot 9$ & $1 \cdot 2$ & $5 \cdot 2$ & 0.4 & $6 \cdot 3$ & $2 \cdot 0$ \\
\hline Depression & $8 \cdot 0$ & 0.0 & $8 \cdot 2$ & 0.5 & $8 \cdot 0$ & 0.0 & $8 \cdot 2$ & 0.4 \\
\hline Vigor & $15 \cdot 5$ & $4 \cdot 1$ & $15 \cdot 10$ & $4 \cdot 3$ & $15 \cdot 3$ & $4 \cdot 2$ & $14 \cdot 0$ & $4 \cdot 1$ \\
\hline Fatigue & $7 \cdot 6$ & $2 \cdot 1$ & 7.9 & 1.8 & 7.5 & 1.5 & 7.9 & 1.8 \\
\hline DSC affective & $15 \cdot 1$ & 0.7 & $15 \cdot 7$ & $1 \cdot 8$ & $15 \cdot 1$ & $1 \cdot 1$ & $16 \cdot 2$ & $2 \cdot 3$ \\
\hline DSC self-devaluative & $14 \cdot 0$ & 0.0 & $14 \cdot 6$ & $1 \cdot 1$ & $14 \cdot 0$ & 0.0 & $14 \cdot 7$ & 1.4 \\
\hline \multicolumn{9}{|c|}{ Recovered depressed ( $n$ 23) } \\
\hline \multicolumn{9}{|c|}{ POMS } \\
\hline Anger & $7 \cdot 5$ & 1.4 & $8 \cdot 7$ & $3 \cdot 0$ & $7 \cdot 2$ & 0.5 & $8 \cdot 3$ & 1.9 \\
\hline Tension & $5 \cdot 4$ & 0.6 & $6 \cdot 8$ & 2.5 & $5 \cdot 4$ & 0.8 & $6 \cdot 8$ & $2 \cdot 8$ \\
\hline Depression & $8 \cdot 2$ & 0.5 & 8.5 & 0.9 & $8 \cdot 1$ & 0.4 & 8.5 & 1.5 \\
\hline Vigor & $11 \cdot 7$ & $5 \cdot 2$ & $10 \cdot 9$ & $4 \cdot 1$ & $12 \cdot 5$ & $4 \cdot 6$ & $11 \cdot 0$ & 3.9 \\
\hline Fatigue & $8 \cdot 2$ & 2.5 & $8 \cdot 8$ & $2 \cdot 4$ & 8.5 & $3 \cdot 2$ & $9 \cdot 1$ & $3 \cdot 4$ \\
\hline DSC affective & $15 \cdot 0$ & 1.0 & $16 \cdot 4$ & $2 \cdot 3$ & $15 \cdot 6$ & $2 \cdot 0$ & $17 \cdot 0$ & $4 \cdot 0$ \\
\hline DSC self-devaluative & $14 \cdot 0$ & 0.2 & $15 \cdot 9$ & 3.4 & $14 \cdot 1$ & 0.5 & $15 \cdot 9$ & $2 \cdot 4$ \\
\hline
\end{tabular}

POMS, Profile of Mood States; DSC, Depressed States Checklist.

For details of diets and procedures, see p. 416 


\section{Discussion}

This study used an $\alpha$-lactalbumin-enriched diet to increase plasma Trp and Trp:LNAA ratio in recovered depressed subjects and controls. We hypothesised that $\alpha$-lactalbumin would have a protective effect against stress, particularly in recovered depressed subjects. Although dietary $\alpha$-lactalbumin led to the expected rises in Trp and Trp:LNAA, only minimal effects were found on mood and cortisol response to stress.

The afternoon values of Trp:LNAA were $73.8 \%$ higher after the $\alpha$-lactalbumin diet than after the casein diet, which is comparable to earlier findings ( $\mathrm{J}$ A J Schmitt et al., unpublished results). In previous studies, increasing Trp:LNAA by $43-48 \%$ (compared with casein) caused a decrease in the cortisol response to stress and reduced depressive feelings under stress in stress-vulnerable subjects (Markus et al. 2000). We found rather small effects of the stressor on cortisol and mood, and these changes were independent of group and diet. These finding are at odds with the experimenters' observations and subjects' comments, which indicated that the procedure was experienced as rather stressful and unpleasant. However, a trend of an order effect was found: $\alpha$-lactalbumin had the expected protective effect on cortisol response if taken on the second day. Also, the change in vigor in response to the stress task was greater on the first day than on the second day, when the casein diet was given. However, no order effects were apparent for all other measures of mood. Nonetheless, the stressor may have been slightly more severe during its first presentation (when subjects were less prepared). It would follow that the protective effects of $\alpha$-lactalbumin were insufficient on the first day.

Markus et al. (2000), using the same stressor, found a protective effect of $\alpha$-lactalbumin on stress-induced changes in mood and cortisol in students with high neuroticism scores. There are a few possible explanations for our contradictory results. First, our recovered depressed subjects may have been 'too healthy'. This group's MADRS scores were below 8, they were not taking antidepressant medication, and they were relatively young, so few previous depressive episodes had occurred. Furthermore, the end of the last episode had been on average a little more than 2 years previously, also suggesting that our patient group was relatively healthy and stable. However, the expected group differences on personality (neuroticism) and markers of depressive vulnerability (cognitive reactivity) were observed, implying that the recovered group was, as a group, more vulnerable than the controls. The mean scores on these scales were above the norms for healthy subjects (Eysenck et al. 1985; Sanderman et al. 1995; Van der Does, 2005) but still not extremely high. Furthermore, this explanation is unlikely because Markus et al. found an effect of $\alpha$-lactalbumin in a probably even healthier group.

A second possible explanation is that the intervention was not strong enough. As will be reported separately elsewhere, $\alpha$-lactalbumin did have an effect on cognitive function in both groups (Booij et al. 2005). These findings suggest that the intervention did not fail, but a $1 \mathrm{~d} \alpha$-lactalbumin diet may be too weak also to affect mood and cortisol responses to stress in recovered patients. Future studies may investigate a higher dose or a longer duration of the diet.

The protective effects of $\alpha$-lactalbumin on cortisol response were also not observed, but this part of the study was hampered by the unexpectedly small effects of the experimental stressor. Previous studies using a different stressor - the Trier Social
Stress-Task - have reported gender differences in cortisol response (Kirschbaum et al. 1999): men show the largest cortisol response, followed by women in their late luteal phase, women in the follicular phase and women taking oral contraceptives. The women in our sample were tested in the late-follicular phase of the menstrual cycle (days 4-10) to minimise possible mood effects. For the purpose of measuring cortisol level, we might better have tested them at a later stage of the cycle. Detailed analyses of gender differences in cortisol response were hampered by the relatively small number of subjects in our sample and by the fact that only five men were included. On the other hand, no gender differences in cortisol response were found in previous studies that used the same stressor as the current study (Markus et al. 2000, 2002).

Another issue often raised in stress research is the influence of context, including the person leading the experiment. In the Trier Social Stress-Task, the people conducting the experiment are instructed to be rejecting and 'unkind' to the participant, adding to the level of stress that is meant to be induced by the task. In our study, however, we used a computerised stress task. The instructions were also all presented by computer. The possibility cannot be excluded that the experimenters in the Markus et al. studies were more 'unkind' to the subjects than in the present study, implying that we may have missed the social component of the stressor.

If both our results and those of Markus et al. are replicable, this implies that neuroticism and a history of depression are not similar concepts in terms of 5-HT vulnerability. Recent studies using the acute tryptophan depletion method found no relationship between neuroticism and the acute tryptophan depletion response (Stewart et al., 2002; L Booij and AJW Van der Does, unpublished results). Another finding from acute tryptophan depletion research is that a depressive response to this manipulation of 5-HT function is mostly found in selective serotonin reuptake inhibitor-treated patients (Delgado et al. 1999). The same may be true for the effects of a Trp-enriched diet. $\alpha$-Lactalbumin possibly has a clearer effect in recovered depressed subjects taking selective serotonin reuptake inhibitors.

In conclusion, $\alpha$-lactalbumin increased plasma Trp and Trp:LNAA in recovered depressed individuals and in healthy controls, but no direct or protective effects were found on mood or on stress-induced cortisol and mood responses. Since cognitive effects did occur (L Booij et al., unpublished results), future studies may investigate the effects of longer-term diets (e.g. $3 \mathrm{~d}$ ) or may investigate different samples (e.g. medicated patients).

\section{Acknowledgements}

All financial and material support for this research are clearly identified in the manuscript. Tryptophan-rich $\alpha$-lactalbumin protein was provided by Friesland Coberco Dairy Foods (Deventer, The Netherlands). None of the authors has financial interests in this manuscript. A. J. W. V. d. D. L. B., R. M. and F. G. Z. participated in the conception and design of the study. W. M. and L. B. participated in the data collection. W. M., A. J. W. V. d. D., R. M. and W. O. participated in the analysis and interpretation of the data. W. O. analysed the amino acid spectra. W. M. and A. J. W. V. d. D. drafted the manuscript. All authors participated in critical revisions of the manuscript. The authors thank Iris Bulthuis, Martine Blok and Ilse van der Meij for assistance with the data collection. 


\section{References}

American Psychiatric Association (1994) Diagnostic and Statistical Manual of Mental Disorders, 4th ed. Washington, DC: American Psychiatric Association.

Attenburrow MJ, Williams C, Odontiadis J, Reed A, Powell J, Cowen PJ \& Harmer CJ (2003) Acute administration of nutritionally sourced tryptophan increases fear recognition. Psychopharmacology 169, 104-107.

Beck AT, Steer RA \& Brown GK (1996) Manual for the Beck Depression Inventory II. San Antonio, TX: Psychological Corporation.

Bell C, Abrams J \& Nutt D (2001) Tryptophan depletion and its implications for psychiatry. Br J Psychiatry 37, 261-270.

Blier P \& De Montigny C (1998) Possible serotonergic mechanisms underlying the antidepressant and anti-obsessive-compulsive disorder response. Biol Psychiatry 44, 313-323.

Booij L, Merens W, Markus CR \& van der Does AJW (2005) Diet rich in a-lactalbumin improves memory in unmedicated recovered depressed patients and matched controls. J Psychopharmacol (In the Press).

Christensen L (1997) The effect of carbohydrates on affect. Nutrition 13, 503-514

Chouinard G, Young SN \& Annable N (1985) A controlled clinical trial of tryptophan in acute mania. Biol Psychiatry 20, 546-557.

Consumer Reports (2004) Readers rate mental-health care for depression. Drug vs. talk therapy: antidepressant effectiveness results and side effects. http://www.infozine.com/news/stories/op/storiesView/sid/3699

Delgado PL, Miller HL, Salomon RM, Licinio J, Krystal JH, Moreno FA, Heninger GR \& Charney DS (1999) Tryptophan-depletion challenge in depressed patients treated with desipramine or fluoxetine: implications for the role of serotonin in the mechanism of antidepressant action. Biol Psychiatry 46, 212-220.

Eysenck SGB, Eysenck HJ \& Barrett P (1985) A revised version of the psychoticism scale. Pers Indiv Diff 6, 21-29.

Fava M (2000) Management of nonresponse and intolerance: switching strategies. J Clin Psychiatr 61, 10-12.

First MB, Spitzer RL, Gibbon M \& Williams JBW (1995) Structured Clinical Interview for DSM-IV Axis I Disorders. (Patient ed. (SCID$I / P)$ ). New York: Biometrics Research Department, NYSPI.

Gallagher DJ (1990) Extraversion, neuroticism and appraisal of stressful academic events. Pers Indiv Diff 11, 1053-1057.

Heine W, Radke M, Wutzke KD, Peters E \& Kundt G (1996) A-lactalbumin-enriched low-protein infant formulas: a comparison to breast milk feeding. Acta Paediatr 85, 1024-1028.

Kendler KS, Kuhn J \& Prescott CA (2004) The inter-relationship of neuroticism, sex, and stressful life events in the prediction of episodes in major depression. Am J Psychiatry 161, 631-636.

Kirschbaum C, Kudielka BM, Gaab J, Schommer NC \& Hellhammer D (1999) Impact of gender, menstrual cycle phase and oral contraceptives on the activity of the hypothalamus-pituitary-adrenal axis. Psychosom Med 61, 154-162.

Maes M \& Meltzer HY (1995) The serotonin hypothesis of major depression. In Psychopharmacology: The Fourth Generation in Progress, pp. 933-944 [FE Bloom and DJ Kupfer, editors]. New York: Raven Press.

Markus CR, Olivier B \& De Haan EHF (2002) Whey protein rich in $\alpha$-lactalbumin increases the ratio of plasma tryptophan to the sum of the other large neutral amino acids and improves cognitive performance in stress-vulnerable subjects. Am J Clin Nutr 75, 1051-1056.

Markus CR, Olivier B, Panhuysen G, et al. (2000) The bovine protein $\alpha-$ lactalbumin increases the plasma ratio of tryptophan to the other large neutral amino acids, and in vulnerable subjects raises brain serotonin activity, reduces cortisol concentration and improves mood under stress. Am J Clin Nutr 71, 1536-1544.

Markus CR, Panhuysen G, Jonkman LM \& Bachman M (1999) Carbohydrate intake improves cognitive performance of stress-prone individuals under controllable laboratory stress. Br J Nutr 82, 457-467.

Markus CR, Panhuysen G, Tuiten A, Koppeschaar H, Fekkes D \& Peters ML (1998) Does carbohydrate-rich, protein-poor food prevent a deterioration of mood and cognitive performance of stress-prone subjects when subjected to a stressful task? Appetite 31, 49-65.

Marsh DM, Dougherty DM, Moeller FG, Swann AC \& Spiga R (2002) Laboratory-measured aggressive behavior of women: acute tryptophan depletion and augmentation. Neuropsychopharmacology 26, 660-671.

McNair DM, Lorr M \& Droppelamn LF (1971) Edits Manual for the Profile of Mood States. San Diego, CA: Educational and Industrial Testing Service.

Meltzer HY \& Lowy MT (1987) The serotonin hypothesis of depression. In Psychopharmacology: The Third Generation of Progress, pp. 513-527 [HY Meltzer and JT Coyle, editors]. New York: Raven Press.

Montgomery SA \& Asberg M (1979) A new depression scale designed to be sensitive to change. Br J Psychiatr 134, 382-389.

Moskowitz DS, Pinard G, Zuroff DC, Annable L \& Young SN (2001) The effect of tryptophan on social interaction in everyday life: a placebocontrolled study. Neuropsychopharmacology 25, 277-289.

Mueller TI, Leon AC, Keller M, et al. (1999) Recurrence after recovery from major depressive disorder during 15 years of observational follow-up. Am J Psychiatr 156, 1000-1006.

Nutt DJ, Forshall S, Bell C, et al. (1999) Mechanisms of action of selective serotonin reuptake inhibitors in the treatment of psychiatric disorders. Eur Neuropsychopharmacol 9, S81-S86.

Ormel J, Oldehinkel AJ \& Brilman EI (2001) The interplay and etiological continuity of neuroticism, difficulties, and life-events in the etiology of major and subsyndromal, first and recurrent depressive episodes in later life. Am J Psychiatry 158, 885-891.

Orosco M, Rouch C, Beslot F, Feurte S, Regnault A \& Dauge V (2004) Alactalbumin-enriched diets enhance serotonin release and induce anxiolytic and rewarding effects in the rat. Beh Brain Res 148, 1-10.

Peters ML, Godaert GL, Ballieux RE, et al. (1998) Cardiovascular and endocrine responses to experimental stress: effects of mental effort and controllability. Psychoneuroendocrinology 23, 1-17.

Sanderman R, Arrindell WA, Ranchor AV, Eysenck HJ \& Eysenck SBG (1995) Het meten van persoonlijkheidskenmerken met de Eysenck Personality Questionnaire (EPQ). Een Handleiding (Measuring Personality Traits Using the Eysenck Personality Questionnaire (EPQ). A Manual). Groningen: Noordelijk Centrum voor Gezondheidsvraagstukken, Rijksuniversiteit Groningen.

Stewart ME, Deary IJ \& Ebmeier KP (2002) Neuroticism as a predictor of mood change: the effects of tryptophan depletion. Br J Psychiatry 181, 242-247.

Teasdale JD \& Cox SG (2001) Dysphoria: self-devaluative and affective components in recovered depressed patients and never depressed controls. Psychol Med 31, 1311-1316.

Van der Does AJW (2001) The effects of tryptophan depletion on mood and psychiatric symptoms. J Affect Disord 64, 107-119.

Van der Does AJW (2002a) Handleiding bij de Nederlandse versie van Beck Depression Inventory-Second Edition (BDI-II-NL) (Manual for the Dutch Version of the Beck Depression Inventory-Second Edition (BDI-II-NL). San Antonio, TX/Lisse, The Netherlands: Harcourt.

Van der Does AJW (2002b) Cognitive reactivity to sad mood: structure and validity of a new measure. Beh Res Ther 40, 105-120.

Van der Does AJW (2005) Thought suppression and cognitive vulnerability to depression. Br J Clin Psychol 44, 1-15.

Wald FDM \& Mellenbergh GJ (1990) De verkorte versie van de Nederlandse vertaling van de Profile of Mood States (POMS) (The Short Version of the Dutch Translation of the Profile of Mood States (POMS)). Nederlands Tijdschrift voor de Psychologie 45, 86-90.

Young SN (1986) The effect on aggression and mood of altering tryptophan levels. Nutr Rev 5, 112-122.

Young SN (1996) Behavioral effects of dietary neurotransmitter precursors: basic and clinical aspects. Neurosc Biobehav Rev 20, 313-323.

Young SN \& Leyton M (2002) The role of serotonin in human mood and social interaction. Insight from altered tryptophan levels. Pharmacol Biochem Behav 71, 857-865. 